\title{
El Convenio Marco para el Control del Tabaco de la Organización Mundial de la Salud
}

\author{
MARISOL ACUÑA A.*
}

The Framework Convention on Tobacco Control of the World Health Organization

The Framework Convention on Tobacco Control (FCTC) is the first international treaty, negotiated under the umbrella of WHO as a coordinated response to the tobacco pandemic, and was unanimously approved by the $56^{\text {th }}$ World Health Assembly in May 2003. It was signed by Chile on september 25, 2003 at United Nations headquarters in New York and it was ratified on june 13, 2005 becoming a law, on september 11, 2005. Since that date the regulatory framework of tobacco in Chile is given by: - The WHO Framework Convention on Tobacco Control (FCTC);• Decree Law 828 (27/12/1978), which regulates issues related to the production and commercialization of tobacco; • Law 19.419 (of 09/09/1995), which regulates advertising, promotion, sponsorship and smoke-free environments, among others. Although our country shows a big legislative production, it can be observed that this one has focused on some specific subjects, important matters are still pending established in the CMTC, in which it is necessary to advance: • Demand reduction measures related to tobacco dependence and cessation;• Tobacco Control Coordinating Committee: • Control of Illicit Trade.

Key words: Tobacco use disorder; tobacco; Pandemics; International cooperation; United Nations.

\section{Resumen}

El Convenio Marco para el Control del Tabaco (CMCT) es el primer tratado internacional, negociado bajo el amparo de la OMS como respuesta coordinada a la pandemia del tabaco y fue aprobado en la $56^{a}$ Asamblea Mundial de la Salud, en mayo de 2003, por unanimidad. Fue suscrito por Chile el 25 de septiembre de 2003 en la sede las Naciones Unidas en Nueva York y ratificado el 13 de junio de 2005 para entrar en vigencia con carácter de ley, el 11 de septiembre de 2005. A partir de esa fecha el marco regulatorio del tabaco en Chile está dado por: - El CMCT; - El Decreto Ley 828 (27/12/1978), que regula temas relacionado con producción y comercialización del tabaco. • La Ley 19.419 (del 6/09/1995), que regula publicidad, promoción, patrocinio y ambientes libres de humo, entre otros. Si bien en nuestro país la legislación para el control del tabaco evidencia una gran producción legislativa, se puede observar que ésta se ha focalizado en algunos temas especificos, quedan pendientes importantes materias establecidas en el CMTC, en las que es necesario avanzar: - Medidas de reducción de la demanda relativas a la dependencia y al abandono del tabaco; • Comisión Coordinadora Control del Tabaco: • Control del Comercio Ilícito.

Palabras clave: Tabaquismo; Tabaco; Pandemia; Cooperación Internacional; Naciones Unidas.

El Convenio Marco para el Control del Tabaco $(\mathrm{CMCT})$ es el primer tratado internacional, negociado bajo el amparo de la Organización Mundial de la Salud (OMS), que busca dar una respuesta coordinada y estructurada a la pandemia del tabaco. Este convenio, cuyo objetivo explícito fue definido como: "proteger a las generaciones presentes y futuras de las devastadoras

* Encargada de Tabaco Ministerio de Salud de Chile (2000-2003 y 2006-2011). Integrante del grupo negociador de Chile del CMCT ante la ONI del Convenio Marco para el Control del Tabaco de la OMS. 
consecuencias sanitarias, sociales, ambientales y económicas asociadas al consumo de tabaco y la exposición al humo de tabaco", fue aprobado en la $56^{\text {a }}$ Asamblea Mundial de la Salud, en mayo de 2003, por unanimidad.

El CMCT aborda por primera vez, todos los factores asociados directa e indirectamente al consumo de tabaco y se establece un lineamiento de acción para que los Estados Parte puedan reducir, de manera continua y sustancial, la prevalencia de consumo de tabaco y la exposición de su población al humo de éste.

El CMCT, entró en vigor el 28 de febrero de 2005, 90 días después que fuera ratificado por 40 estados. Actualmente cuenta con 180 Estados Parte que han ratificado el tratado, entre éstos, nuestro país.

\section{Breve Historia}

El tabaco, es un producto de origen vegetal, elaborado a partir de la planta nicotiniana tabacum, oriunda de América, que se caracteriza por tener un alto contenido de Nicotina en sus hojas. Aunque desde el siglo XVII, se hicieron reiterados intentos por controlar el consumo del tabaco, a través de la incorporación de leyes y regulaciones, estas medidas fracasaron, en especial a partir de fines del siglo XIX, cuando por diversos factores, entre los que destaca la industrialización de la producción de cigarrillos, el consumo de éstos se expandió.

Desde mediados del siglo XX, se hizo cada vez más evidente la asociación causal entre el consumo de tabaco y la exposición a su humo, con el desarrollo de enfermedad y muerte. A fines del siglo pasado, el tabaco llegó a ser considerado la principal causa de enfermedad y muerte evitable a nivel global, con una mortalidad estimada por sobre los 5 millones de muertes por año. En la década del 90, el profesor Peto y cols, en conjunto con el Banco Mundial ${ }^{1}$, proyectaron que, de mantenerse las condiciones existentes, entre 1950 y 2050 habrán muerto 530 millones de personas a causa del tabaco, muchos de ellos en ese momento, niños, adolescentes y no fumadores expuestos al humo de tabaco ajeno.

En este escenario y considerando la complejidad y multiplicidad de factores asociados, la OMS decidió impulsar una estrategia innovadora: un acuerdo internacional, que estableciera un marco de acción para "proteger a las generaciones presentes y futuras de las devastadoras consecuencias asociadas al consumo de tabaco". Este acuerdo, es lo que hoy conocemos como el $\mathrm{CMCT}^{2}$.
Este tratado se organiza en un Preámbulo y 38 artículos, que conforman once Partes, cada una de éstas dedicada a un tema específico. Desde el primer párrafo, el CMCT declara que las Partes en ese Convenio "están determinadas a dar prioridad a su derecho de proteger la salud pública"3.

\section{Los 10 años de CMCT en Chile}

Chile suscribió el CMCT de la OMS, el 25 de septiembre de 2003 en la sede las Naciones Unidas en Nueva York. Luego, lo ratificó el 13 de junio de 2005 y entró en vigor con carácter de ley, el 11 de septiembre de 2005.

A partir de esa fecha el marco regulatorio del tabaco en Chile está dado por:

- El CMCT.

- El Decreto Ley 828 (27/12/1978), que regula temas relacionado con producción y comercialización del tabaco.

- La Ley 19.419 (del 6/09/1995), que regula publicidad, promoción, patrocinio y ambientes libres de humo, entre otros.

Durante estos 11 años de vigencia del CMCT en nuestro país, se han promulgado leyes orientadas a adecuar el marco legal del control tabaco a los requerimientos del tratado. El proceso se inició con la Ley 20.105, que modificó a la ley 19.419, que entró en vigor en agosto de 2006 y dio así inicio al proceso su implementación en Chile. La primera advertencia gráfica en los productos de tabaco entró en vigor en noviembre de ese año. Desde entonces, se ha continuado avanzando en su cumplimiento, desarrollando leyes y decretos que modifican tanto a la ley 19.419 como al decreto-ley $828^{4}$.

\section{Un compromiso pendiente}

El CMCT representa "el conjunto de medidas que como mínimo se deben aplicar para lograr el adecuado control del tabaco". Esto implica que para reducir los niveles de consumo y exposición al tabaco se requiere cumplir y desarrollar como línea de base, todas las estrategias y/o líneas de acción incluidas en el convenio; se entiende que la omisión de alguna de éstas debilita el impacto de las estrategias ya aplicadas, y pone en riesgo el logro esperado.

Si bien nuestro país evidencia una gran producción legislativa, se puede observar que ésta se ha focalizado en algunos temas específicos, quedando todavía pendientes importantes materias 
establecidas en el CMTC, en las que es necesario avanzar. Entre éstas es pertinente destacar:

- Medidas de reducción de la demanda relativas a la dependencia y al abandono del tabaco. El artículo 5-2b, establece para cada Parte, la obligación de elaborar políticas apropiadas para prevenir y reducir el consumo de tabaco, la adicción a la nicotina y la exposición al humo de éste. En el artículo 14, el tratado establece en forma perentoria que cada Parte deberá incorporar el diagnóstico y tratamiento de la dependencia del tabaco y también servicios de asesoramiento para el abandono del consumo.

A este respecto es posible afirmar que nuestro país muestra los índices de consumo de tabaco más altos de la región, y no hay un programa estructurado a nivel nacional de apoyo a la cesación, se conocen sólo experiencias aisladas, no coordinadas, no comparables, ni oficialmente validadas.

- Comisión Coordinadora de Control del tabaco: Artículo 5, Obligaciones generales, 2 letra a). Éste establece que cada Parte deberá establecer o reforzar y financiar un mecanismo coordinador nacional o centros de coordinación para el control del tabaco. Hoy, es de suma relevancia, mejorar el nivel de coordinación entre los todos los actores involucrados en el control del tabaco, incluyendo las áreas gubernamentales de Salud, Educación, Hacienda, Economía, y Sociedad Civil.

- Control del Comercio Ilícito: El artículo 15, aborda el comercio ilícito de cigarrillos y productos de tabaco, lo que es de sumo interés para nuestro país. En febrero de 2016 se promulgó la ley 20.899, que establece una nueva normativa para la implementación de un sistema de trazabilidad para los cigarrillos producidos e importados, autorizados para ser comercializados en Chile. El Reglamento para implementar el sistema de trazabilidad, debió ser publicado en agosto del presente año, y transcurridos 5 meses aún no se ha publicado. Cabe destacar que en noviembre de 2012, la Conferencia de las Partes, adoptó el "Protocolo para la eliminación del comercio ilícito de tabaco"s, definido como un tratado independiente complementario al CMCT, que fue negociado por los Estados Partes del Convenio y que tendrá vigencia solo para aquellos que lo ratifiquen. Este Protocolo está actualmente a la espera de ser adoptado por un número de Partes suficientes para entrar en vigor.

A la fecha de este informe, aún no ha sido firmado por nuestro país y dada la relevancia del tema, parece conveniente sugerir que las autoridades competentes consideren su suscripción y posterior ratificación.

Desde la entrada en vigencia del CMCT de la OMS, se han generado en nuestro país cambios evidentes, tanto en el comportamiento de la población en cuanto al respeto a los ambientes libres de humo, al consumo de tabaco en escolares y adultos jóvenes ${ }^{6}$, como también a los que se refieren a la publicidad y advertencias sanitarias en los envases. Sin embargo, parece urgente y necesario evaluar objetivamente los logros alcanzados, avanzar en los temas pendientes e identificar las necesidades actuales para readecuar las estrategias a la nueva realidad y seguir progresando para lograr el control de la epidemia del tabaco y la reducción de sus nefastas consecuencias en la salud de la población de nuestro país.

\section{Bibliografía}

1.- La epidemia de tabaquismo: Washington, D.C.: -OPS, C1999, pág. 93 Disponible en: www.paho.org

2.- Información sobre CMTC disponible en http://www. who.int/fctc/es/

3.- La Tabla de Contenidos del CMTC, se estructura del siguiente modo: Prefacio; Preámbulo; Parte I: Introducción; Parte II: Objetivos, principios básicos y obligaciones generales; Parte III: Medidas relacionadas con la reducción de la demanda del tabaco; Parte IV: Medidas relacionadas con la reducción de la oferta de tabaco; Parte V: Protección del medio ambiente; Parte VI: Cuestiones relacionadas con la responsabilidad; Parte VII: Cooperación técnica y científica y cooperación de información; Parte VIII: Arreglos institucionales y recursos financieros; Parte IX: Solución de controversias; Parte X: Desarrollo del Convenio; parte XI: Disposiciones finales.

4.- Modificaciones a estas normativas en http://www.bcn.cl

5.- Ver protocolo en http://apps.who.int/iris/bitstre am/10665/80881/1/9789243505244_spa.pdf

6. Estudios de Consumo de Población general y escolar http://www.senda.gob.cl/observatorio/estudios/

Correspondencia a:

Dra. Marisol Acuña A.

Email: SOLACUNA2006@gmail.com 